\title{
Carnets
}

Revue électronique d'études françaises de l'APEF

Deuxième série - 13 | 2018

Corps, rythmes et voix : en/jeux littéraires et artistiques

\section{Entre tradition et transgression,}

La poétique du conte dans l'œuvre de Michel Tournier

\section{Arlette Bouloumié}

\section{CpenEdition}

\section{Journals}

Édition électronique

URL : http://journals.openedition.org/carnets/2586

DOI : $10.4000 /$ carnets.2586

ISSN : 1646-7698

Éditeur

APEF

Référence électronique

Arlette Bouloumié, «Entre tradition et transgression, 》, Carnets [En ligne], Deuxième série - 13 | 2018,

mis en ligne le 31 mai 2018, consulté le 02 mai 2019. URL : http://journals.openedition.org/

carnets/2586 ; DOI : 10.4000/carnets.2586

Ce document a été généré automatiquement le 2 mai 2019.

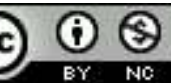

Carnets est mis à disposition selon les termes de la licence Creative Commons - Atribution - Pas d'utilisation commerciale 4.0 International. 


\title{
Entre tradition et transgression,
}

\author{
La poétique du conte dans l'œuvre de Michel Tournier
}

\section{Arlette Bouloumié}

1 J'ai souvent invité Michel Tournier dans mes classes, du lycée à l'université, où il aimait dialoguer avec les étudiants. Il avait une aisance extraordinaire à l'oral. Dans ses déplacements à l'étranger, au Sénégal en particulier, à l'invitation du Président Senghor, il ne manquait jamais d'aller échanger avec les jeunes collégiens ou lycéens. Dans un entretien pour la revue Europe, en 1989, à l'occasion de la publication de son recueil de contes et nouvelles, Le Médianoche amoureux, il dit: «Avant d'écrire une histoire, je la répète une dizaine de fois autour de moi, quand je vais dans une école, quand je fais une conférence. (...) La répétition est création.» (Europe, 1989: 148-149). Ce en quoi il se rapproche de la tradition orale. Pour situer Tournier dans cette opposition de l'oral et de l'écrit, je citerai ces lignes qu'il écrit en 1994 dans Le Miroir des idées (Tournier, 2001) ${ }^{1}$ intitulées « La parole et l'écriture »:

La parole est première. Dieu créa le monde en le nommant. C'est le Verbe créateur. L'écriture qui apparut des millénaires plus tard découle de la parole, et a besoin d'elle pour l'irriguer. Toute l'histoire de la littérature est faite de retours constants de l'écriture à cette source vive et vivifiante qu'est le langage parlé. Un grand auteur est celui dont on entend et reconnaît la voix dès qu'on ouvre l'un de ses livres. Il a réussi à fondre la parole et l'écriture. (MI : 111-112)

2 Tournier cite les contes comme les plus hauts sommets de la littérature mondiale. «Je ne place rien au-dessus du Chat Botté, du Petit Poucet de Perrault » dit-il et le premier chapitre de son autobiographie, Le Vent Paraclet ${ }^{2}$, contient un éloge de La Reine des neiges d'Andersen, ce conte «qui associe la familiarité la plus quotidienne et le fantastique le plus grandiose » (VP: 50-52). Il précise dans un article du Monde de l'Éducation: «Les contes remontent à la tradition la plus ancienne puisque les Mille et une nuits et les Paraboles de l'Évangile relèvent de ce genre à la fois bref, débordant de significations, mais mystérieux et chiffré. » (Tournier, 1998).

3 L'évolution de Tournier du naturalisme mystique à la féerie du conte a surpris ceux qui avaient été séduits par ses romans. Le Coq de Bruyère, publié en 1978 est le premier recueil de contes et nouvelles, avant Le Médianoche amoureux ${ }^{3}$ (1989), témoignant d'une évolution vers un idéal de concision, de limpidité et de brièveté. Tournier reprend les thèmes des 
contes traditionnels disparus, ceux de la mythologie populaire qui s'adressent aux enfants mais tout autant aux parents. De cette technique de la réécriture Tournier affirme : «Peut-être le comble de l'art consiste-t-il à créer du nouveau en lui prêtant un air de déjà vu qui rassure et lui donne un retentissement lointain dans le passé du lecteur ", ce qui crée une indispensable complicité entre donneur et receveur (VP, 205). Il favorise en même temps une double lecture, celle de l'enfant et celle de l'adulte. Gérard Genette lui fait écho dans Palimpsestes:

Disons (...) que l'art de « faire du neuf avec du vieux » a l'avantage de produire des objets plus complexes et plus savoureux que les produits «faits exprès»: une fonction nouvelle se superpose et s'enchevêtre à une structure ancienne, et la dissonance entre ces deux éléments coprésents donne sa saveur à l'ensemble. (Genette, $1982: 451$ )

Nous verrons d'abord la définition des contes selon Tournier avant d'en étudier quelquesuns, présents dans son premier recueil Le Coq de Bruyère, à savoir : «La Fugue du petit Poucet » reprise d'un texte de Perrault, puis «Tristan Vox» qui reprend un mythe plus général, celui du double, inspiré du romantisme allemand, enfin, «La famille Adam», réécriture d'un texte de la Genèse, et, dans Le Médianoche amoureux, un conte dans la tradition orale des Mille et une nuits et des contes orientaux.

Tournier place en exergue, au-début du Coq de Bruyère, trois vers de Lanza del Vasto extraits du Chiffre des choses (1942):

Au fond de chaque chose, un poisson nage.

Poisson, de peur que tu n'en sortes nu,

Je te jetterai mon manteau d'images.

Dans la dixième des Lettres parlées à son ami allemand Hellmut Waller $(2015)^{4}$, il déclare :

Ces trois vers je les aime beaucoup parce qu'ils définissent toute une esthétique, le conteur étant celui qui ne veut pas que les poissons qui sont au fond des choses apparaissent dans leur nudité effrayante et incompréhensible. Ça, c'est le métaphysicien. Le conteur, lui, leur jette son manteau d'images, c'est-à-dire qu'il les habille avec sa propre substance, à lui conteur, sa vérité subjective. » (LP : 139)

Le conte a pour lui une signification philosophique cachée comme la parabole.

Remarquons que le conte, chez Tournier, n'est pas toujours publié seul, il est souvent enchâssé comme dans La Goutte d'or, roman réaliste où deux contes, «Barberousse » et "La Reine blonde », illustrent les pouvoirs tantôt bienfaisants tantôt destructeurs de l'image. Ces contes s'insèrent parfois dans le texte lui-même comme dans le conte de Noël «La Fugue du Petit Poucet » où le récit de la Chute des hommes dans le règne animal de la violence est fait par Logre, héros du conte qui devient conteur lui-même. Dans Le Médianoche amoureux, ce sont les nouvelles puis les contes, racontés par les invités, dont dépend le dénouement du roman, à savoir si les époux restent ensemble ou se séparent. Les contes dialoguent avec le récit-cadre, l'éclairent, illustrant ici la fonction de réconciliation de la littérature.

9 Tournier souligne, dans Le Vol du Vampire (1981)5, la proximité du conte et du mythe: « Noyés dans l'épaisseur d'une affabulation puérile [...] travestis et brisés, (les mythes) n'en prêtent pas moins leur puissante magie à une historiette populaire.» (VV: 43). Or qu'est-ce qu'un mythe ? C'est « un édifice à plusieurs étages » (VP:188) dont le rez-dechaussée est enfantin et le sommet métaphysique. Il se prête ainsi à une double lecture pour les enfants et pour les adultes. 
10 Dans l'article «Barbe-Bleue et le secret du conte» (VV: 38), Tournier compare la nouvelle, la fable et le conte. La nouvelle qui se rapproche du fait divers dans les journaux, relate une « histoire vraie » souvent triste et dépourvue de message. La fable, elle, comporte un enseignement, non de morale, mais de sagesse, mélange d'intelligence et d'expérience. Quant au conte, à «l'épaisseur glauque», il est "hanté par une signification fantomatique qui nous touche, nous enrichit mais ne nous éclaire pas » (VV: 40); c'est un "souvenir vague et insaisissable [...] de réminiscence, comme l'ombre du souvenir » $(V V: 42)$ qui explique son attrait. La nouvelle, souvent pessimiste et dépourvue de transcendance, de même que la fable, trop didactique, intéressent moins Michel Tournier que le conte, cette "nouvelle hantée " qui nous livre souvent, selon lui, une vision plus optimiste. Selon Jean-Bernard Vray : «La nouvelle serait le pur constat d'une réalité morose et même sordide. Le conte, au contraire, se caractériserait par une plénitude spirituelle cachée faite d'échos et de réminiscences. » (Vray, 1997 : 107)

11 Michel Tournier puise dans les traditions les plus diverses, il se qualifie lui-même de « pie voleuse ». Il y a, dit-il, une "appropriation du conteur qui ne fait que préparer une seconde appropriation celle de l'auditeur» (Europe, 1989 : 149). Tantôt il réécrit un texte précis : celui de Charles Perrault dans «La Fugue du Petit Poucet », celui de Victor Hugo dans Angus, reprise de "L'Aigle du Casque » de La Légende des siècles, ou encore Pierrot ou les secrets de la nuit qui s'inspire de la commedia dell'arte, de même, dans le conte « L'âne et le bœuf " de Gaspard, Melchior \& Balthazar (1980) ${ }^{6}$, l'âne savant, Kadi Chouia, renvoie à Cadichon, le héros des Mémoires d'un âne de La comtesse de Ségur (GMB : 162); tantôt le conte s'inspire d'un mythe populaire comme celui de l'ogre ou du double issu du romantisme allemand (Chamisso ou Hoffmann) avec les contes : "Tristan Vox », "Que ma joie demeure », «Le nain rouge ». Ou bien c'est la réécriture ludique d'un passage de la Bible, notamment sur la création de l'homme dans « La Famille Adam » du Coq de Bruyère $(1978)^{7}$, « Le Sosie de Dieu » dans Le Vagabond immobile et « La Légende de la musique et de la danse » dans Le Médianoche amoureux. Ou bien encore, la réécriture prend la forme d'un conte oriental inspiré des Mille et une nuits comme « Barberousse » et « La reine blonde » dans La Goutte d'or, ou "Les deux banquets ou la commémoration », dans Le Médianoche amoureux; dans ce même recueil, le texte intitulé « La Légende de la peinture » puise, lui, dans la tradition persane.

Si le titre de cette communication commence par : "Entre tradition et transgression ", c'est que Michel Tournier se livre dans ces contes à une imitation caractérisée par une inversion ironique, parodique, impliquant une distance critique.

Nous prenons comme premier exemple «La Fugue du petit Poucet». Ce conte est une réécriture poétique et humoristique du célèbre conte de Perrault. La nuit de Noël, Pierre Poucet fuit la demeure de son père, le Commandant Poucet, chef des bûcherons de Paris, pour se retrouver dans la forêt où le rencontrent les filles de Monsieur Logre. Dans ce conte écologique, la forêt devient un refuge, loin de la ville et des écheveaux d'autoroutes. Ce Logre est végétarien et il déteste la chasse et la violence. C'est le Commandant Poucet qui incarne la violence, lui qui a détruit les arbres de Paris. Tournier multiplie les inversions paradoxales liées à l'actualisation du conte dans le monde contemporain. L'auteur a en effet rencontré, lors d'un voyage au Canada, un groupe de hippies qui, en 1970, étaient à la une de l'actualité. Ils rejetaient les valeurs conventionnelles de la société de consommation et voulaient créer une contreculture pacifique, en contact avec la nature. L'attirance qu'ils exerçaient sur les jeunes qui fuguaient inquiétait les adultes. Logre séducteur est ici un détourneur aux yeux de la 
société. Mais ce "géant des bois » délivre aussi, en cette fête sacrée de Noël, une parole poétique de célébration: «Écoutez-moi ! Le Paradis, qu'est-ce que c'était? C'était une forêt. » (CB : 59) Se reportant au monde des origines comme dans les mythes, il évoque la sagesse de l'arbre cosmique, "cette calme croissance dans une union de la terre et du soleil » et le passage du règne végétal au règne animal, nouvelle version de la Chute originelle. Il se fait conteur à l'intérieur du conte. La juxtaposition des différents points de vue, celui de l'enfant qui admire, celui du Commandant Poucet qui accuse, celui de Logre lui-même qui prend la parole, contribue à l'ambivalence du conte et l'indécidabilité du sens. Le réalisme satirique pour décrire le monde moderne coupé de la nature n'exclut pas la poésie liée à l'évocation du monde sacré des origines dans le discours de Logre. Enfin la technique de la réminiscence qui caractérise le conte selon Tournier est abondamment illustrée dans ce texte. Derrière Logre se profilent l'androgyne mythique de l'âge d'or, aux longs cheveux blonds et à la voix fluette qui monte jusqu'aux trilles les plus aigües, mais aussi le Père Noël qui donne en cadeau des bottes de rêve à l'enfant ; et aussi le Christ au moment de son arrestation lorsque le Commandant Poucet arrête Logre; enfin, le conteur lui-même, Michel Tournier, assis près de la cheminée, dans un grand fauteuil d'osier, un "véritable trône, mais un trône léger, aérien, adorné de boucles, de ganses, de croisillons » ( $C B: 56)$, où l'on reconnaît le sien quand il reçoit ses hôtes. Le paysage et les lieux sont d'ailleurs ceux des environs de Choisel où habitait Michel Tournier : la forêt de Rambouillet, le Christ de Saclay. On reconnaît la voix de l'auteur et sa présence, en filigrane, dans l'humour lié à la présentation constante des choses sous un jour inattendu, dans cette association du réalisme satirique et de la poésie cosmique, dans le jeu des réminiscences, la présence du conte dans le conte, et la double lecture de l'adulte et de l'enfant.

Sans reprendre un texte précis comme c'est le cas dans «La Fugue du petit Poucet », le conte intitulé "Tristan Vox» illustre le mythe du double associé au problème de l'identité. Autant que le mythe de l'ogre, celui du double a nourri l'œuvre de Tournier comme dans les contes «Que ma joie demeure » et «Le Nain rouge » que je n'aborderai pas aujourd'hui. Le mythe du double trahit l'influence du romantisme allemand. Ce texte est lié à l'expérience personnelle de l'auteur, à ces années de travail à la Radio diffusion nationale puis à Europe 1. C'était l'époque où la télévision n'existait pas encore :

Pour rester sans visage ni regard, les voix n'en avaient que plus de mystère et leur magie agissait avec une efficacité parfois redoutable sur les hommes et les femmes à l'écoute. On remarquera que dans nombre de religions, les décrets de Dieu se manifestent par une voix tombant $d u$ haut du ciel vide. Aussi les spiqueurs paraissaient-ils au grand public comme des créatures incorporelles et douées d'ubiquité, à la fois toutes puissantes et inaccessibles. Certains prenant la parole tous les jours à la même heure [...] jouissaient d'une notoriété extraordinaire. ( $C B$ : 125)

Des rapports très mystérieux s'établissaient alors entre le spiqueur invisible et les auditeurs, expérience qui rejoint celle du conteur et ce bonheur de l'oralité que Michel Tournier cherche à faire passer dans ses contes.

Félix Robinet est victime du surgissement d'un double triomphant, quasi divin : à partir de sa voix, transfigurée par la magie du micro, des milliers d'auditeurs construisent un personnage imaginaire à la séduction irrésistible, Tristan Vox. L'opposition des prénoms, Félix et Tristan, souligne l'opposition du personnage réel, "gris et paisible ", et du héros romantique prestigieux que la foule imagine: « un homme grand, mince [...] avec une masse de cheveux indomptés », un masque tourmenté «malgré la douceur de ses grands 
yeux mélancoliques ». L'antithèse est totale entre "ce héros ténébreux » et le père tranquille bedonnant qui lui a donné naissance par sa voix: "Comment expliquer la magie de cette voix ? Il y avait en elle une gravité caressante et veloutée que relevait une fêlure, une cassure, quelque chose de blessé et qui blessait aussi avec une implacable douceur. » $(C B: 126)$. Le nom très prosaïque de Robinet accentue encore l'opposition avec " cette voix d'or » ( $C B: 129)$ qui s'adresse « aux yeux de l'âme et non à ceux du corps » ( $C B$ $: 126)$ et qui bouleverse les foules. Pourtant, lorsque Félix était acteur, elle n'avait bouleversé personne. Sa femme même lui avoue qu'elle ne la reconnaissait pas. Notons que la voix est ici transfigurée par la technique de l'enregistrement. Tournier fait un usage audacieux de la pensée de Hegel : puisque les Idées sont des êtres vivants, il suffit que l'Idée - ici le personnage imaginaire de Tristan Vox - prenne une force suffisante pour qu'elle substitue sa nécessité à la contingence du monde sensible. Ce personnage imaginaire, fécondé et chargé de réalité par les rêves de milliers d'auditeurs, va s'incarner et c'est l'homme réel qui va devenir «l'ombre et comme la doublure de l'Autre » (CB : 131), illustrant la victoire du mythe sur la réalité, idée chère à Michel Tournier.

Félix Robinet se rend bien compte qu'il y a dans ce double quelque chose qui menace son existence. Il est « enfoui dans une sorte de tombeau » quand «Tristan Vox retentissait comme un dieu omniprésent [...] s'introduisait dans les cœurs, se déployait en Phénix resplendissant » $(C B: 134)$. Une erreur dans un journal donne à Tristan Vox le visage de Frédéric Durâteau, « finaliste de la Coupe Borotra du tennis-club de Nanterre » ( $C B: 138)$, qui ne tarde pas à se présenter à Félix. Tous les personnages se sentent vaciller dans leurs certitudes, un vent de folie passe: la secrétaire, Mademoiselle Flavie se jette par la fenêtre, Amélie, la femme de Félix, laisse brûler ses cailles. Car ces deux femmes qui sont les seules à connaître la double identité de Félix Robinet sont trompées par ce double idéal, chargé d'une énergie positive énorme. Le dédoublement de Félix entraine celui de sa femme qui, sous le nom d'Yseult, cherche à reconquérir cet inconnu, son mari qui, sous le nom de Tristan Vox, devient l'amour imaginaire d'une foule de femmes. L'épouse sage devient la maitresse à l'érotisme déchaîné. Yseult à son tour se dédouble. Mademoiselle Flavie, la vieille fille "raide et sombre comme la justice" se met à écrire des lettres " criardes et obscènes " pour obliger Tristan Vox à se manifester car comment justifier une vie consacrée à un être qui n'existe pas? À l'hôpital le double monstrueux de Mademoiselle Flavie apparaît aux yeux stupéfaits de Félix Robinet : c'est un personnage nouveau, enturbanné de crêpe blanc et barbouillé d'ecchymoses violacées, un " mamamouchi au visage tuméfié » ( $C B: 144)$, «à la face de clown blafarde et bariolée » ( $C B: 146)$. Avec le thème du clown, nous avons ici l'exemple d'une réalité tragique perçue de manière bouffonne. Félix décide d'interrompre les émissions mais il surprend bientôt sa femme écoutant à la radio "la voix chaude, juvénile et sympathique » de Frédéric Durâteau. L'apparition du double rejette le moi dans le néant après l'avoir fait douter de sa propre existence.

On peut se demander si ce texte est véritablement un conte ou une nouvelle. On y part de la réalité, mais le texte s'élève au fantastique par l'intervention du mythe du double et de celui de Tristan et Yseult. Le fantastique est ici lié au phantasme collectif d'individus victimes de leurs désirs et de leurs rêves. Le mythe de Tristan et Yseult, même traité sur un mode parodique, contribue à faire décoller le récit du réel et à le charger d'un sens étrange et inquiétant qui le rapproche du conte. Le héros vit la découverte de son double comme une crise d'identité douloureuse. Mais le narrateur, lui, s'amuse - non sans une pointe de cruauté - de ces « loufoqueries du destin» $(C B: 129)$ qui font sortir Tristan Vox 
de Félix Robinet, par le simple truchement du micro comparé à « un serpent électronique hostile et méchant» (CB: 133), qui opérait une «métamorphose [...] pas moins mystérieuse que celle d'une citrouille en carrosse par un coup de baguette magique » ( $C B$, 129) et nous retrouvons ici l'univers des contes de Perrault.

La suite des péripéties et des rebondissements, avec les dédoublements en série, relève d'un comique de la farce que ne renierait pas Molière. Michel Tournier dessine ses personnages à grands traits mordants, caricaturaux, cocasses. Le comique vient surtout du décalage entre le mythe de Tristan et Yseult, mythe de l'amour fou et idéalisé, et les aspirations très "pot-au-feu» de Robinet qui touche à une "béatitude toute gastronomique " $(C B: 131)$ devant les "petits plats, les plus canailles de la cuisine bougnate » que lui concocte avec amour sa femme. L'humour lié à un mélange de réalisme et de fantastique dédramatise un récit qui se termine mal puisque Tristan Vox, supplanté par son double, perd son travail.

20 Troisième exemple où l'on retrouve le ton parodique et l'écart humoristique : « La famille Adam» qui ouvre Le Coq de Bruyère, réécriture cette fois d'un texte sacré, celui de la Genèse. Le conte commence par opérer une déduction logique de l'affirmation biblique : «L'homme a été fait à l'image et à la ressemblance de Dieu.» Ce qui entrâne en conséquence l'androgynie d'Adam.

21 Les relations des personnages sublimes, Adam et Jéhovah, sont ici celles d'un fils avec son père, ramenées à des dimensions humaines familières. Jéhovah voudrait être grand-père mais Adam résiste; Jéhovah crée alors le Paradis. Adam étant toujours mécontent, Jéhovah le coupe en deux, opération de modelage qui aboutit à la création d'Eve. La deuxième partie se situe après qu'Adam et Eve ont été chassés du Paradis. Caïn se consacre à l'agriculture; Abel, nomade comme son père, se plaît à détruire son œuvre. Caïn exaspéré finit par lui "casser la tête ", expression familière qui ramène la scène tragique à une simple dispute. Chassé par Jéhovah, il crée une ville avec un temple où Jéhovah finit par s'installer, fatigué d'être «cahoté à dos d'homme sur une Arche d'Alliance vermoulue qui puait le suint de bélier» ( $C B: 17)$. Le Dieu terrible de l'Ancien Testament n'est plus qu'un vieillard entêté, bien content de trouver assistance auprès de celui qu'il a chassé : Caïn.

Bakhtine définit le carnavalesque comme l'abolition des distances au profit d'un contact libre et familier :

L'objet d'une représentation sérieuse est peint sans aucune distance épique ou tragique, est donné non pas dans le passé absolu d'un mythe ou d'une légende mais au niveau du présent, dans la zone d'un contact direct et familier avec des contemporains vivants (Bakhtine 1970 : 152).

23 Le style oral, familier, souligne l'abaissement carnavalesque: "Caïn souriait dans sa barbe », «Jéhovah [est] toujours un peu grognant pour la forme » ( $C B: 18)$.

L'inversion de la tradition est patente : Caïn est réhabilité et présenté comme la victime d'Abel et le premier héros civilisateur, architecte, agriculteur. L'ironie est liée à la liberté iconoclaste de l'inversion, à cet aspect subversif qui bouscule les idées reçues. Contrairement au dogme chrétien de la Chute, Tournier montre la fécondité de l'erreur : la Chute est créatrice puisqu'elle suscite l'esprit d'invention. Au-delà de l'aspect ludique et de l'humour, le conte fait l'éloge du génie créateur de l'homme. Ce conte peut paraître sacrilège en ce qu'il montre les conséquences positives exceptionnelles de ce qui est d'ordinaire présenté comme une catastrophe sans retour. 
le cas des contes qui illustrent la tradition orientale impliquant la présence chaleureuse de l'auditoire. Le conte oriental se prête à ces imitations puisque, oral à l'origine, il vit des variantes que lui imposent les différents conteurs confrontés à leur public. Tournier réintroduit l'ouverture rituelle : «il était une fois » et met en scène les conteurs comme Sangali qui s'adresse aux rois mages dans le conte «Barbedor" de Gaspard, Melchior \& Balthazar et qui rappelle Shéhérazade par la menace qui pèse sur lui lorsqu'Hérode lui dit : «Si tu ne me fais pas rire, tu ne sortiras d'ici qu'essorillé. » (GMB: 109) ; ou bien c'est, dans La Goutte d'or (1986) ${ }^{8}$, le conteur noir, Abdullah Fehr, venu des confins du Sahara et du Tibesti pour raconter «Barberousse » $(G O: 31)$, et aussi le maître calligraphe Abdel Al Ghafari qui invite le héros Idriss «avec quelques autres [...] à s'asseoir autour de lui pour entendre la légende de la Reine blonde» (GO: 203). Tournier aimait recréer cette situation chaleureuse, conviviale, lorsqu'il allait dans les écoles. Dans un texte inédit intitulé "Un nouveau venu en librairie, le livre-à-écouter», il s'enthousiasme :

Ils m'écoutent, ils m'écoutent, accroupis sur leurs talons, drapés dans leur boubou, les yeux rieurs et la bouche entr'ouverte sur leurs dents blanches, les enfants sénégalais, gabonais, togolais, béninois. "Il était une fois une reine, un calife d'Ispahan, un petit pêcheur de Douarnenez, un Ogre tapis dans la forêt profonde. » Je fonctionne comme un conteur. Je me prends pour un griot, une Shéhérazade. Je puise à pleines mains dans tous les folklores, ajoutant de plein droit mon jus, $m a$ voix, mes sucres et mes acides, mes caresses et mes coups. Hier à Madras, demain à Abou Dhabi. Si je le pouvais, ce n'est pas roman que j'écrirais sur la couverture de mes livres, ce serait conte. Mais qui comprendrait? (archives de l'auteur; projet d'article non publié)

Dans «La légende de la peinture » du Médianoche amoureux (MA : 294-298), le narrateur, écrivain-conteur comme Tournier, se voit proposer par un ami un procédé nouveau de diffusion massive de ses œuvres. Pour illustrer l'idée que la «communication ajoute à la création une vie innombrable et imprévisible sans laquelle elle n'est qu'un objet inerte ", le narrateur conte à son ami « la parabole du sage derviche Algazel, plus justement appelé Rhazali ou Ghazali, un peu arrangée [...] comme il est loisible de le faire dans la tradition orale » (MA : 296) :

Il était une fois un calife de Bagdad qui voulait faire décorer les deux murs de la salle d'honneur de son palais. Il fit venir deux artistes, l'un d'Orient, l'autre d'Occident. Le premier était un célèbre peintre chinois qui n'avait jamais quitté sa province. Le second, grec, avait visité toutes les nations (...). Ce n'était pas qu'un peintre. (...) Le calife leur expliqua son propos et confia à chacun l'un des murs. (MA :296)

Le Chinois demande un délai de trois mois pour achever sa fresque. Questionné par le calife, le Grec répond : «Quand mon confrère chinois aura terminé, j'aurai terminé. » Un rideau divise la pièce en deux pour éviter que les artistes ne se gênent. Trois mois plus tard la cour, « cortège magnifique » avec des « robes brodées, panaches de plumes, bijoux d'or, armes ciselées » se rassemble d'abord du côté du mur peint par le Chinois : «Ce ne fut qu'un cri d'admiration. La fresque figurait en effet un jardin de rêve planté d'arbres en fleurs avec des petits lacs [...] qu'enjambaient de gracieuses passerelles. Une vision paradisiaque. » Beaucoup de spectateurs pensèrent le Chinois vainqueur avant même de voir l'œuvre du Grec. «Le calife fit tirer le rideau qui séparait la pièce en deux. [...] La foule se retourna et laissa échapper une exclamation de stupeur émerveillée " (MA : 298). Le Grec n'avait rien peint. Il avait installé un miroir qui couvrait le mur et reflétait le jardin du Chinois, mais ce jardin était animé par une foule magnifique de gens qui 
«bougeaient, gesticulaient et se reconnaissaient avec ravissement » et le Grec fut déclaré vainqueur, à l'unanimité. «La légende de la peinture est tirée de la tradition persane » ( Europe, 1989 : 149) déclare Michel Tournier. Ce récit est effectivement la réécriture d'un texte extrait de l'Anthologie du soufisme d'Eva de Vitray-Meyerovitch (éd. Sindbad, 1978: 38-39), intitulé "L'apologue des peintres de Byzance et de Chine», parabole racontée, après Ghazâlî au XI ${ }^{\mathrm{e}}$ siècle, par le mystique persan Rûmi dans Mathnavî (I, 3467 et suiv.) au XIII ${ }^{\mathrm{e}}$ siècle. J'ai retrouvé dans les manuscrits de Michel Tournier la photocopie de ces deux pages du livre d'Eva de Vitray-Meyerovitch. Dans ses entretiens pour la revue Europe, Tournier commente ce texte énigmatique :

Le seul fait que la peinture du Chinois se reflète dans le miroir arrive à hausser cette peinture à un degré supérieur. L'idée c'est que l'image est supérieure à la chose. L'image de l'image est supérieure à l'image. C'est subtil. Mais j'ai apporté mon grain de sel. La supériorité de la peinture reflétée dans le miroir c'est que la cour était là, alors que dans le conte originel, le paysage était désert (Europe, 1989 : 149-150).

Notons que le mot légende, dans le titre définitif, est employé à la place du mot conte. Tournier, dans ses notes manuscrites, définit la légende d'après le latin legenda, « chose devant être lue», "récit fabuleux, généralement édifiant. Texte d'explication accompagnant une image ». C'est que ce conte énigmatique (du miroir) est enchâssé dans le texte pour illustrer une thèse: la complémentarité de la création et de la communication comme le montre, dans le manuscrit, le titre premier du récit : « Les deux amis ou création et communication ». Que serait en effet un livre sans son lecteur, une pièce de théâtre sans son metteur en scène, une histoire sans le conteur qui la raconte à son public? Le message n'était pas le même à l'époque de Rûmi. La «Légende de la peinture » se rapproche de la parabole, récit allégorique contenant un enseignement, du mot grec parabolé qui signifie comparaison. Dans ce conte, les moyens technologiques modernes de diffusion valorisent la communication.

Le Médianoche amoureux, dans son ensemble, peut être considéré comme un éloge de la parole vivante des conteurs puisqu'il s'adresse à tout un auditoire. Un couple qui ne sait plus communiquer veut annoncer la mauvaise nouvelle de leur séparation à tous ses amis. Il les invite à un médianoche où on parlera d'amour. Ça doit durer toute la nuit et chacun raconte une histoire comme dans le Décaméron de Boccace au xive siècle. Ce sont d'abord des nouvelles, tristes, qui contribuent à aggraver la séparation du couple, mais au cours de la nuit il y a un renversement. Il y a trois ou quatre nouvelles dont on ne sait pas si ce sont des contes ou des nouvelles. Puis des contes, savoureux, chaleureux, affables et finalement le couple décide de ne plus se séparer. Le Médianoche suggère donc une fonction de réconciliation de la littérature : «Si les nouvelles s'étaient imposées d'abord par leur vérité pesante et mélancolique, les contes avaient gagné au fur de la nuit en beauté et en force pour atteindre un rayonnement d'un charme irrésistible.» (MA: 47) Les époux concluent: "Ce qui nous manquait c'était une maison de mots où habiter ensemble $\gg(M A: 48)$.

Dans un entretien, Tournier compare ce pouvoir de la parole du conteur à celui de Shéhérazade qui, sans poignard comme Thésée mais avec ses contes va désarmer le calife, vrai Minotaure, qui veut changer de femme tous les jours : « La littérature peut empêcher les tyrans de faire des massacres. Il faut leur raconter des histoires » (Europe, juin-juillet $1989: 150)$. 
31 À la fin du Médianoche, les légendes de la musique et de la danse, comme celle des parfums, célèbrent la grandeur du génie humain qui triomphe du malheur de la Chute en inventant les arts dont la fonction apparaît dès lors comme une recherche du Paradis perdu, vision optimiste et stimulante car le temps qui détruit tout ne détruit pas la musique, pas la peinture, pas la sculpture, pas la poésie.

Le dernier conte, «Les deux banquets ou la commémoration ", qui conclut le recueil Le Médianoche amoureux, confirme cet optimisme et fait écho à la thématique du récit-cadre sur les relations du couple et le rôle qu'y jouent la routine et la répétition. C'est une réponse lumineuse. Il relate le concours culinaire organisé par le calife d'Ispahan pour désigner un nouveau chef cuisinier. Les deux cuisiniers en compétition présentent, à une semaine d'intervalle, le repas de leur création. Le premier emporte l'adhésion de tous par ses qualités mais le second surprend, proposant un repas identique à celui de son concurrent. C'est pourtant celui-ci que le calife désigne comme vainqueur, car «le premier banquet était un événement, mais le second était une commémoration » (MA : 302), démontrant ainsi le caractère sacré de la répétition, ce que Tournier explique par un exemple : «Dans les Évangiles, les juifs se réunissent le jeudi soir pour la Cène. Ce n'est pas l'Eucharistie, c'est la Pâque juive. On est en plein judaïsme (...) Ce n'est qu'après sa mort [du Christ] dans les Actes des apôtres que les apôtres se réunissent pour partager le pain et le vin et qu'est née l'Eucharistie » (Europe, op.cit., 1989 : 150)

L'auteur défend aussi, indirectement, ces genres de la répétition que sont le conte et le mythe, au service de la célébration.

Le conte écrit garde donc la marque de la tradition orale par un style parlé familier, fréquemment utilisé par Tournier, par les variantes qu'il apporte aux textes figés dont il s'inspire et ce goût de la transgression parfois provocatrice où se manifeste la présence du conteur, cet humour, cette volonté de faire rire qui aide à surmonter le pessimisme et à garder la distance avec les conformismes. Tournier écrit : "C'est cela un conte : un formidable questionnement caché sous un masque enfantin de guignol et de comédie italienne.» (« Petits lecteurs, grands textes », Le Monde de l'éducation, Paris, mars 1998).

\section{BIBLIOGRAPHIE}

BAKHTINe, Mikhaïl (1970). La Poétique de Dostoïevski. Paris : Seuil.

Europe (1989). «Rencontre avec Michel Tournier », Europe, n 722-723, juin-juillet, p. 148-149.

GENETTE, Gérard (1982). Palimpsestes, La littérature au second degré. Paris : Seuil.

TOURNIER, Michel [1980]. Gaspard, Melchior \& Balthazar. Paris : Gallimard, « Folio », 2011.

TOURNIER, Michel [1981]. Le Vol du Vampire. Paris : Gallimard, « Folio », 1994.

TOURNIER, Michel [1986]. La Goutte d'or. Paris : Gallimard, « Folio », 1987.

TOURNIER, Michel [1977]. Le Vent Paraclet. Paris : Gallimard, « Folio », 1997.

TOURNIER, Michel (1998). « Petits lecteurs, Grands textes », Le Monde de l'Éducation, mars. 
TOURNIER, Michel [1994]. Le Miroir des idées. Paris : Gallimard, « Folio », 2001.

TOURNIER, Michel [1989]. Le Médianoche amoureux. Paris : Gallimard, « Folio »,2003.

TOURNIER, Michel [1978]. Le Coq de Bruyère. Paris : Gallimard, « Folio », 2005.

TOURNIER, Michel (2015). Lettres parlées à son ami allemand Hellmut Waller (1967-1998). Paris :

Gallimard.

VRAY, Jean-Bernard (1997). Michel Tournier ou l'écriture seconde. Lyon : Presses Universitaires de Lyon.

\section{NOTES}

1. Abrégé MI.

2. Abrégé $V P$.

3. Abrégé MA.

4. Abrégé $L P$.

5. Abrégé $V V$.

6. Abrégé $G M B$.

7. Abrégé $C B$.

8. Abrégé $G O$.

\section{RÉSUMÉS}

Nous nous proposons d'étudier chez Michel Tournier la réécriture des contes populaires de la tradition orale (Perrault) et ceux inspirés du romantisme allemand, de la Bible, des Mille et une nuits. Nous étudierons la définition que Tournier donne du conte en l'opposant à la nouvelle et à la fable, ses liens étroits avec le mythe.

Les constantes de la réécriture: inversion, actualisation, carnavalisation, mélange des tons, inquiétante étrangeté ou comique, poésie ou réalisme, usage de la réminiscence, double lecture, nature du fantastique, seront mis en valeur dans les contes étudiés.

Dans chaque texte, la présence en filigrane, pleine d'humour, du conteur, traduit l'importance que Tournier accorde à la tradition orale et au message philosophique sous-jacent.

Les textes étudiés sont, dans Le Coq de Bruyère : "La Fugue du Petit Poucet ", "Tristan Vox », «La famille Adam», et, dans Le Médianoche amoureux : «La légende de la peinture » et «Les deux banquets ou la commémoration ».

We want to study, in Michel Tournier's works, the rewriting of popular tales of the oral tradition (Perrault) and those inspired by German romanticism, or by the Bible or One Thousand and one nights. We shall give Tournier's definition of the tale in opposition with the novel and his analogy with the myth.

The characteristics of the rewriting: inversion, actualization, disturbing quaintness or comic, poetry or realism, use of reminiscences, double reading, nature of fantastic, will be studied. In each text, the presence, full of humour, of the teller, shows the importance for Tournier of the oral tradition and of the philosophical message. 
The texts studied are, in Le Coq de Bruyère: "La Fugue du Petit Poucet", "Tristan Vox", "La famille Adam", and in Le Médianoche amoureux: "La légende de la peinture" and "Les deux banquets ou la commémoration".

INDEX

Keywords : tale, myth, oral tradition, rewriting, philosophy

Mots-clés : contes, mythe, tradition orale, réécriture, philosophie

\section{AUTEUR}

\section{ARLETTE BOULOUMIÉ}

Université d'Angers

bouloumi[at]@club-internet.fr 